\title{
Full-Wave Modeling of THz RTD-Gated GaN HEMTs
}

\author{
Sai N. Tenneti, Student Member, IEEE, Niru K. Nahar, Senior Member, IEEE, and John L. Volakis, Fellow, IEEE \\ ElectroScience Laboratory, Electrical and Computer Engineering Dept., The Ohio State University, \\ 1330 Kinnear Road, Columbus OH, 43212, USA
}

\begin{abstract}
Modeling transistors at terahertz frequencies is challenging, because electromagnetic and quantum effects that are negligible in lower frequencies become limiting factors in device performance. Though previous work has focused on modeling the channel of a high-electron mobility transistor (HEMT) using hydrodynamic equations, a more complete toolset is needed to describe submillimeter-wave device gain performance. This paper introduces a simulator that couples fullwave Maxwell's equations with Schrodinger-based charge transport equations, and is used to evaluate the gain performance of a GaN HEMT at THz. This novel simulator is also used to evaluate the effect on gain when a resonant tunneling diode (RTD) is integrated with a HEMT. Upon validation with published work, we state the feasibility of RTD-gated GaN HEMT structures that have resonances up to $2.3 \mathrm{THz}$ and gain up to $6 \mathrm{~dB}$.
\end{abstract}

Index Terms - Field effect transistors, HEMTs, Resonant tunneling devices, THz devices

\section{INTRODUCTION}

$\mathrm{H}$ IGH-POWER TERAHERTZ DEVICES are needed for communications, imaging, radar, and radio astronomy applications [1]. However, achieving good gain performance at $\mathrm{THz}$ frequencies has been a challenge, especially for silicon-based devices, though research on silicon-based lasers as $\mathrm{THz}$ sources is ongoing [2]. Nevertheless, there has been a strong interest [3-7] to explore various materials and structures to improve performance at higher frequencies.

To increase the frequency of operation, heterostructures with high electron mobility at material interfaces [3-7] have been considered. These have increased transconductance that leads to improved gain at higher frequencies. Indeed, recent work [8-10] demonstrated that simple design modifications, such as those of placing resonant tunneling diodes (RTDs) at the gate, can minimize losses and increase the resonance and gain of HEMTs. This performance improvement was demonstrated using distributed models that implement hydrodynamic equations in Agilent's Advanced Design System (ADS) software, shown in Fig. 1. These hydrodynamic equations [11-14] model electron drift in HEMT channels as plasma waves. The latter can then be used to explain the increased gain amplification. However, to validate this explanation, it is important to employ electromagnetic and semiconductor device models that can be fabricated and measured.

Conventional device simulation tools tend to be limited by the standard quasi-static models in their toolsets. But the latter become inaccurate at $\mathrm{THz}$ and $\mathrm{mm}$-wave frequencies. This is because the conductivity effects and other parameters (e.g. potentials, fields, and densities) need be modeled as functions of frequency with no simplified assumptions. Therefore, electromagnetic full-wave tools are required to properly model $\mathrm{THz}$ and sub-THz transistors, including HEMTs. Indeed, fullwave modeling methods such as finite-difference time domain (FDTD) techniques have already been used [15-22] to model transistor devices such as HEMTs and MESFETs. In this case, full-wave equations were coupled with electron transport equations via hydrodynamic [15-16] or Monte Carlo iterations [17-18]. However, these hybrid full-wave approaches have only been tested up to $80 \mathrm{GHz}$. Furthermore, the semiclassical transport equations do not account for interference effects and rapidly varying fields in heterojunctions, which are increasingly significant at microscopic scales. To account for these effects, which are transverse to the direction of transport, quantum corrections need to be added to the modeling toolset. Therefore, conventional full-wave modeling needs to be augmented with Schrodinger's relation.

In this work, a simulation model is proposed to couple fullwave equations with Monte Carlo (MC) iterations, while concurrently incorporating Schrodinger-based corrections. As an example application, our model is specialized for a $\mathrm{THz}$ GaN HEMT and RTD-Gated GaAs and GaN HEMT, shown in Fig. 1. These are then compared with previously published results, particularly with those in [9] and [19] for validation.

Both of these devices (RTD and HEMT) operate on certain phenomena that can be explained by theoretical equations, such as hydrodynamic theory and negative differential conductance. These supposedly occur in devices with short

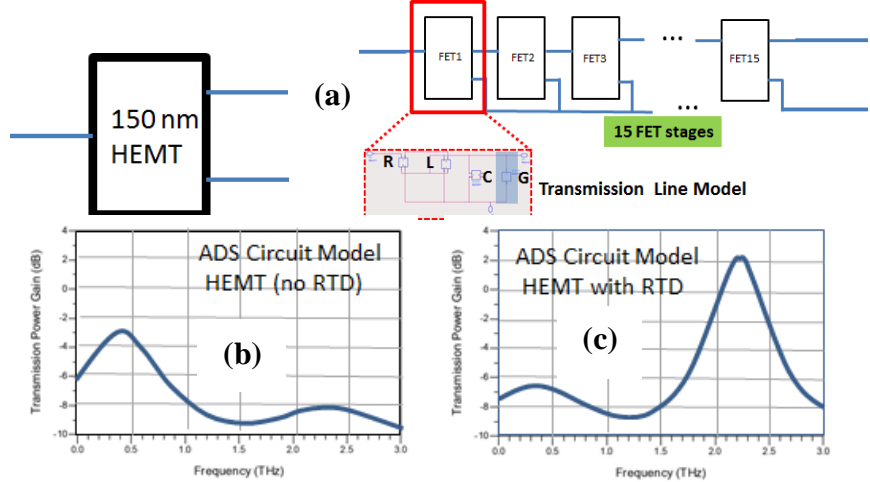

Fig. 1. (a) Generic ADS Schematic of a HEMT with gate length of $150 \mathrm{~nm}$. This device is composed of 15 cascaded stages, each with $10 \mathrm{~nm}$ gate length and represented with a transmission line model [9] (b) HEMT predicted gain performance (c) Predicted HEMT gain performance when RTD is integrated. A gain increase and resonance shift occurs with this integration. 
gate lengths and confined channel regions. Before going into details on the device simulation technique, some background on previous work regarding the developed theory will help to understand the work.

\section{Distributed CiRCuIt MOdel for RTD-HEMT}

Previous researchers have proposed that modifying the channel properties of a HEMT and adding negative differential conductance (NDC) layers can potentially improve its responsivity at $\mathrm{THz}$ frequencies. Firstly, in [11] was developed the hydrodynamic theory for semiconductors, which explains the movement of electrons confined within the HEMT channel behaving as a two-dimensional electron fluid. Stated in [12] was that detector responsivity could be enhanced in HEMTs when the gate is connected to a structure that exhibits NDC properties. These explanations come from the understanding of plasma wave behavior, as the characteristic frequency of the plasma wave falls in the $\mathrm{THz}$ regime for a typical sheet charge density in a nanoscale HEMT. However, at the same time, theoretical and experimental studies [9-13] have shown that gate leakage to be a key parasitic that reduces detector responsivity. The gate leakage increases the plasma wave damping, representing a positive gate conductance. In order to properly reduce the damping, the gate conductance needs to be counteracted with a negative conductance, which the resonant tunneling diode provides. This in turn can lead to significant stable power gain at $\mathrm{THz}$ frequencies.

This phenomenon is best described with a distributed circuit model of the HEMT channel, and in its basic form as shown in Fig. 1. This model in its most general form is a transmission line (TL) model developed in [13], specifically to analyze electron plasma-wave based HEMTs. The HEMT channel is simply cascaded stages of this transmission line. Each of the transmission line parameters were explicitly defined in terms of the distributed channel resistance, kinetic conductance, presented as a function of frequency. Without the NDC (no G in the TL model), there is no power gain. However, it is possible to achieve greater than $4 \mathrm{~dB}$ gain when the appropriate source-load impedances are chosen, and the conductance is added. These gain results portray the ideal situation of the performance we desire for the RTD-gated HEMT. Our work presented in this paper and also previously in [8], has focused on modeling a realistic device model that matches these gain curves. We believe this approach provides confidence that a device exhibiting the mentioned plasmonic properties can be structurally realizable. Subsequent sections explain our development of these device structures, along with our validations with theoretical results from [9-10].

\section{SEMICONDUCtor Modeling OF RTD-HEMT StRUCTURE}

Among the available modeling softwares, commercial electromagnetic simulators using finite-element method (implemented in ANSYS HFSS), and electronic device simulation toolsets (implemented in Silvaco Atlas) were used in this work. Silvaco Atlas has the capability of modeling semiconductor devices in $2 \mathrm{D}$ or $3 \mathrm{D}$. It can be used to interface

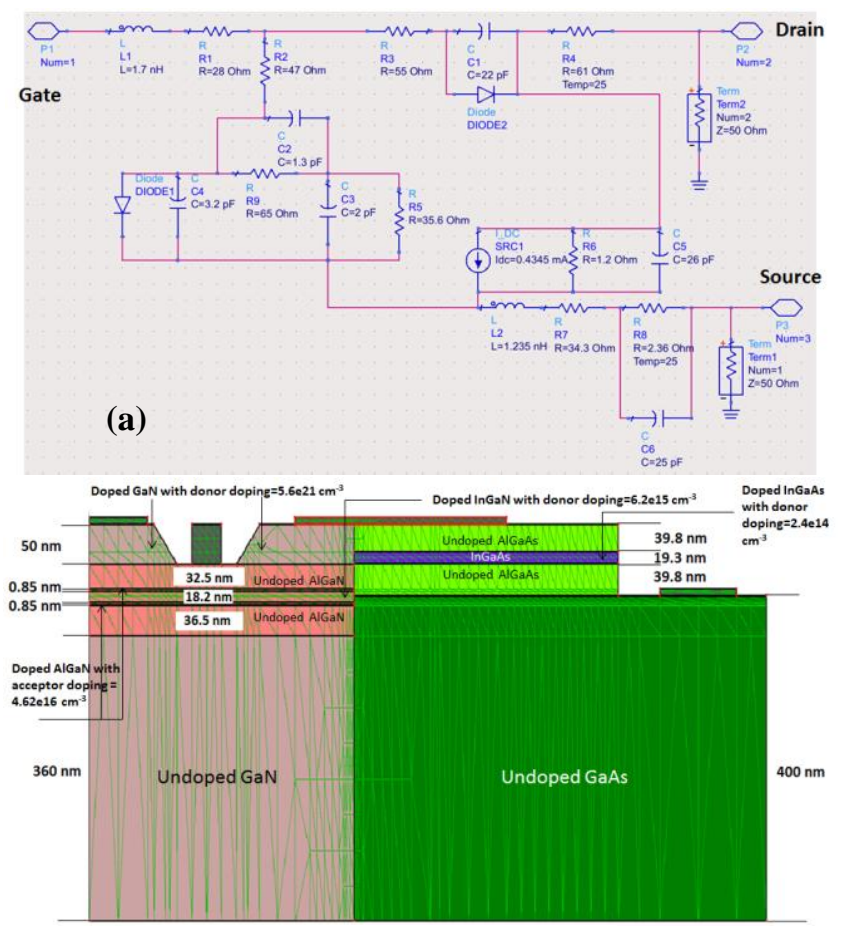

(b)

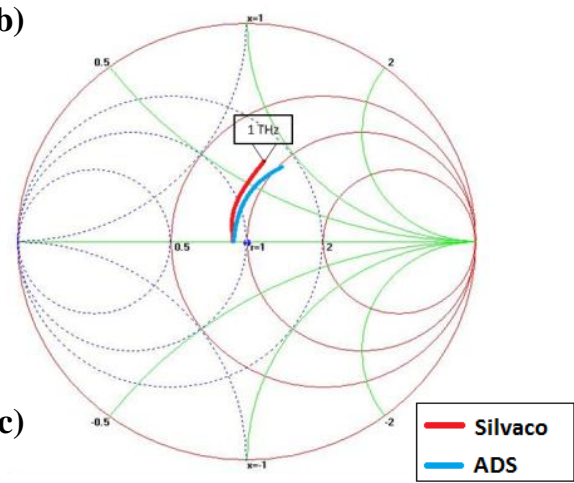

Fig. 2. (a) Equivalent Circuit Model of AlGaN/GaN HEMT (b)

2. Comparison of S21 between ADS (circuit) and Silvaco (device) models. The optimized dimensions of the device model are shown.

with other CAD softwares, also I-V and C-V curves are used to extract intrinsic device parameters, such as $V_{t}, g_{m}$, and $I_{D S}$.

As it is well known, the foundations of Silvaco Atlas are Poisson's equation, the carrier continuity equation, and constitutive equations which are approximations of the Boltzmann Transport equation (ex. drift-diffusion model, energy balance equation, hydrodynamic model). Mobility is an important parameter for the constitutive equations, and Silvaco gives several options to model the mobility (as function of charge concentration, temperature, or E-field components). With the different combinations arising from the available equations, Atlas can solve up to six coupled PDEs. The mesh, PDEs, and discretization procedure will determine the non-algebraic problem to be solved. This also depends on how much coupling exists between the equations of use. Silvaco offers the Gummel (decoupled), Block (partiallycoupled), and Newton (fully coupled) solution techniques for discretization. Even though Silvaco Atlas has a vast set of equations to model intrinsic effects of the device, it cannot model device interactions with the surrounding environment 
(interference/coupling effects), because the electromagnetics equations are not developed within. Initially, we aimed to model the RTD-gated HEMT structure with Silvaco Atlas. As reference data was easily available for GaAs-based RTDs, the initial investigation started out with evaluating the performance of a GaN HEMT when integrated with a GaAs RTD. Because of lattice mismatches between the GaAs-based RTD and GaN HEMT, the two structures needed to be interconnected horizontally as shown in Fig. 3, rather than grown together vertically on one substrate. The distributed models we used based from [9-10], and the Silvaco schematic are both shown in Fig. 2.

When optimizing the parameters of the device dimensions as well as the doping values for the semiconductor layers, a perceptible design was obtained that matched the expected theoretical S21 performance of the circuit model. However, it was quickly realized that the semiconductor equations inherent in the toolset did not take dispersion and frequency variations into account when we aimed to model devices operating at sub-mm wave frequencies. Therefore, a new method was needed that accounted for the electromagnetic wave effects occurring at such high frequencies. Full-wave implementations taking into account the complete electromagnetic and quantum effects at the heterojunctions showed to be more useful.

\section{FULL-WAVE MODELING OF RTD-HEMT STRUCTURE}

The GaN HEMT and GaAs RTD structures are geometrically modeled and simulated as shown in Fig. 3. A FDTD code was also developed based on the method

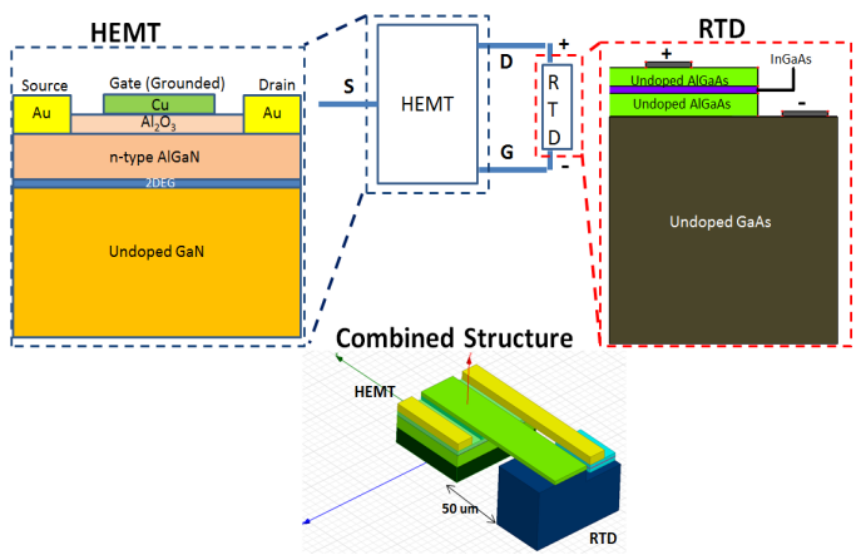

Fig. 3. Schematic of a GaN HEMT integrated with a GaAs RTD.

presented in [20], to accurately characterize the field propagation. Compared to the frequency domain in which the modeling of active properties is difficult, modeling in FDTD solves Maxwell's equations coupled with the active device model in complete form. While this is ongoing, the field values $\mathrm{E}$ and $\mathrm{H}$ are updated at every time step, assuming uniform, linear, and isotropic relations for the permittivity and permeability. $\mathbf{J}$ is updated as the conductivity is modified using the transport equations. Specifically, FDTD was used to model the transistor structure with boundary conditions as in [21] (PMC on four sides, PEC at the bottom, absorbing boundary conditions at top, for both structures). The simulation domain of the HEMT is depicted in Fig. 4. The full-wave model operates with a step size of $1 \mathrm{~nm}$, and time step of $0.1 \mathrm{ps}$. The potential profile of the channel before and after applied bias is plotted for proof of concept. Fig. 5 and Fig. 6 show examples of charge velocity and field profiles as a

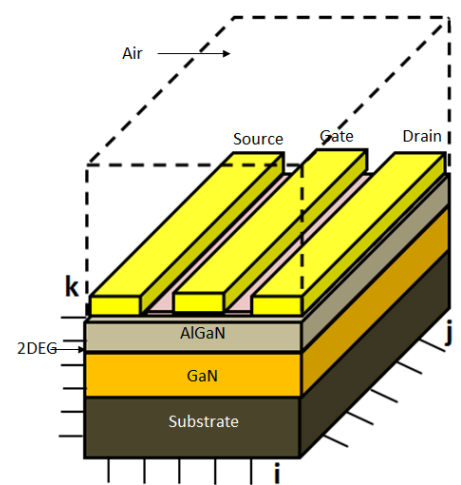

Fig. 4. FDTD Schematic for Simulation for ordinary AlGaN/GaN HEMT operating at $\mathrm{THz}$, with layers and boundary region shown

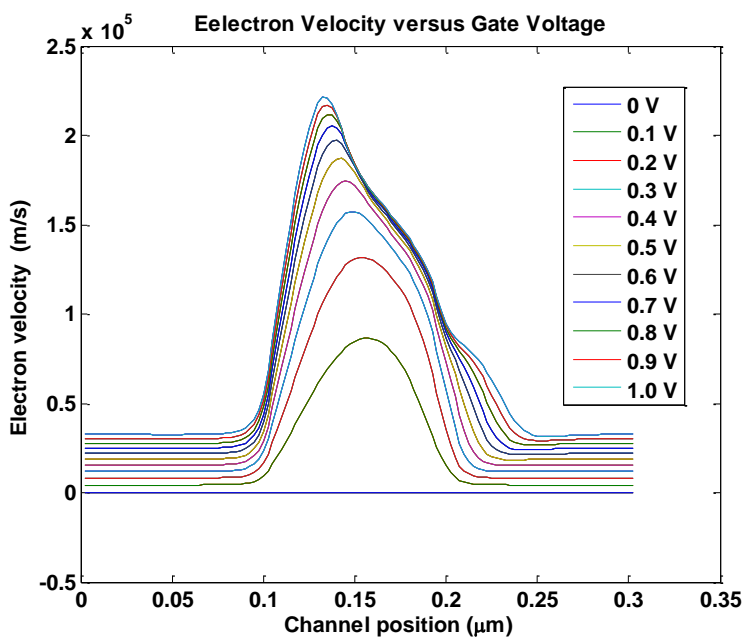

Fig. 5. Charge velocity as a function of applied bias for HEMT channel
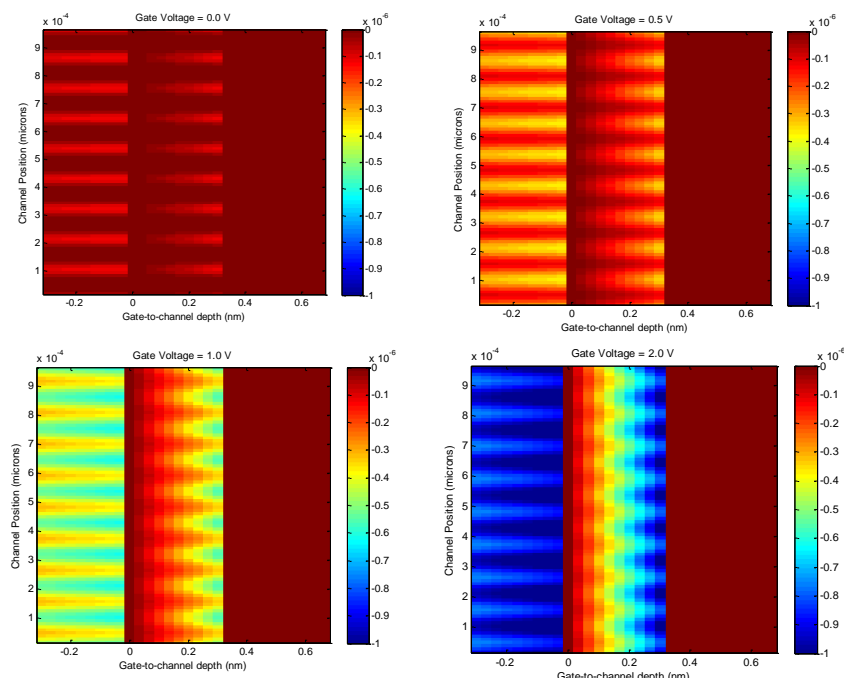

Fig. 6. Impact of DC Voltage on Field Profile. Plotted for Gate Voltage $=0 \mathrm{~V}$, $0.5 \mathrm{~V}, 1 \mathrm{~V}$, and $2 \mathrm{~V}$ 
function of applied bias, respectively. These guarantee the convergence of the solutions and help in making important design decisions for the dimensions, necessary doping, and bias. The 2D electron gas (2DEG) layer [22] was also incorporated into the FDTD model. As the 2DEG channel region is significantly thinner than the rest of the layers, an adaptive gridding scheme was implemented to improve the modeling accuracy of the 2DEG channel. An appropriate distance of $50 \mu \mathrm{m}$ was also placed between the GaAs RTD and GaN HEMT to ensure their functionality remains independent where required.

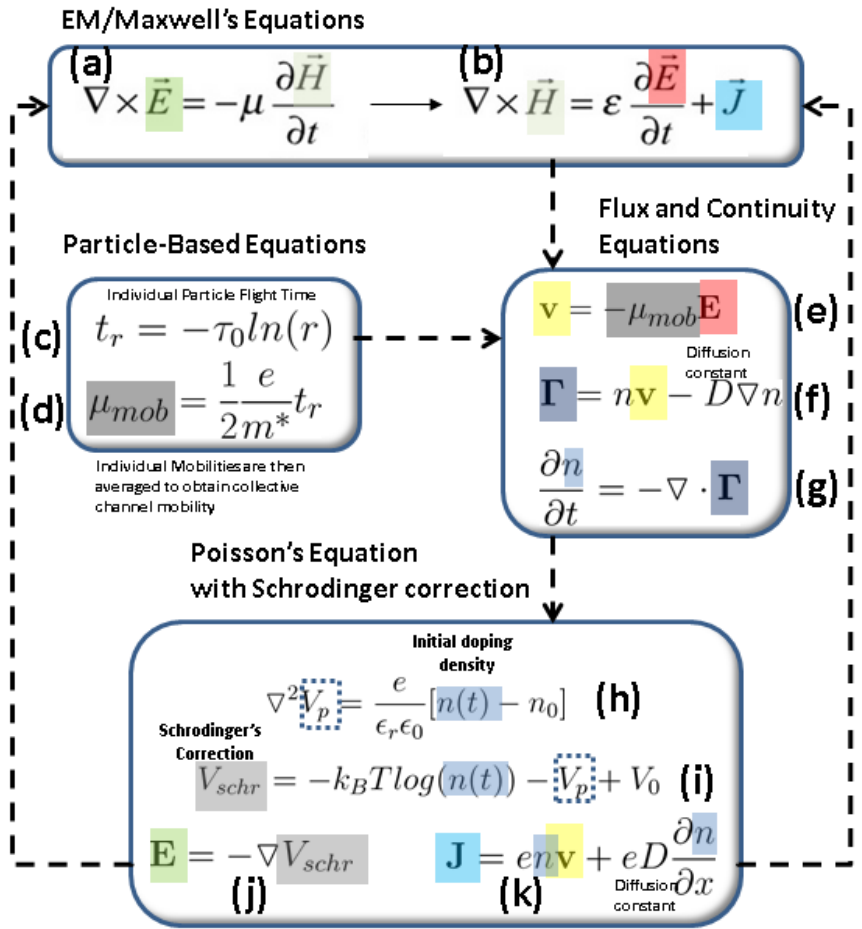

Fig. 7. Full-Wave/Transport Equation Set. Same colored parameters indicate that the newly iterated value are fed to the next equation where used.

The electromagnetic solver is useful to calculate field variations due to the moving charges in the $2 \mathrm{D}$ electron gas channel. The electron transport simulator then updates charge properties altered by the applied fields. If physical parameters such as fields and the current density are updated iteratively between the two models, an appropriate link is established between the simulators. To carry out the simulations, an input signal of amplitude $0.1 \mathrm{~V}$ between 0.1 and $3 \mathrm{THz}$ was introduced at the source terminals at discrete frequencies. Using this biasing input signal, Maxwell's equations were used to determine the potentials at each and every point of the device. Subsequently, the processes in Fig. 7 (a) and (b) were used to update the electric and magnetic fields sequentially, as done in conventional FDTD implementations. However, accurate modeling of the 2DEG requires the introduction of transport equations to describe the electron dynamics using Boltzmann relations. This portion also included the accounting for the quantum effects at the heterojunction.

\section{2DEG ModELING}

The hybrid model that we developed consists of an integration of particle-based equations, continuity equations, Poisson's, Maxwell's, and Schrodinger's equations. Before the particle-based self-scattering equations are evaluated, initial bias conditions are set based on the $\mathrm{dc}$ and ac source applied between the source and gate terminals. From the initial bias conditions, the initial fields can be calculated and in turn the potential distribution for the entire structure is found. With the help of a simple hydrodynamic equation, $\mathrm{n}_{\mathrm{s}}$ the sheet charge density is calculated along the channel. This gives an initial account for the number of electrons along the channel.

The randomized flight time is calculated for each particle individually using a random number generator, with the help of equation 7 (c). This is the first step in calculating the trajectories of the individual particles. From the flight time, the mobility is calculated for each individual particle using 7 (d) and then averaged across the entire channel. The electron drift velocity is then calculated in 7 (e) using the mobility value and the converged E-field from the full-wave equations. Using this drift velocity, flux is calculated in 7 (f) and the charge profile is subsequently updated with the continuity equation in $7(\mathrm{~g})$.

Most importantly, for $\mathrm{THz}$ frequencies, quantum corrections are needed to account for the quantum repulsion effect in the heterojunction (e.g. $\mathrm{AlGaN} / \mathrm{GaN}$ ) that results in quantum subbands [24]. The Schrodinger correction takes into account the abrupt changes in field occurring at the heterojunction, and further improves the accuracy of the model. As no major differences are observed at higher subband levels and to limit unnecessary complexity, just one subband level is considered in the calculations. To start the iterations, $\mathrm{V}_{0}$ is set to a value such that $\mathrm{V}_{\text {schr }}=0 . \mathrm{T}$ is a transverse temperature based on data presented in [24], and $k_{B}$ is Boltzmann's constant. The charge density and classical, uncorrected potential calculated from Poisson's equation in 7 (h), are fed into the quantum correction equation in 7 (i) to obtain a new corrected potential. The E-field and electric current density are subsequently updated and fed back into Maxwell's equations to determine new fields using equations 7 (j) and (k). This process continues until $\mathrm{E}$ and $\mathrm{H}$ reach convergence to within $\delta<$ 0.001 , i.e. within 3 decimal places of accuracy. The output potential at the drain is found using the final field values. Comparison of the output vs. input voltage values as well as currents derived from the $\mathrm{J}$ values, determines the device power gain. This process is repeated for several frequencies, from 0 to $3 \mathrm{THz}$, to ultimately determine the transistor gain.

For our simulations, we found that the gain was comparable to the results in [9], which includes electron drift in the HEMT channel using the hydrodynamic equations presented in [11]. However, our proposed approach includes Schrodinger's effects and charge fluctuations that may be significant at $\mathrm{THz}$, whereas the hydrodynamic model approximates these parameters. 
Fig. 8 shows a comparison of the full-wave FDTD (without electron mobility equations) and our proposed hybrid fullwave model with Schrodinger's equations. The gain vs. frequency curves for these two full-wave solutions are compared with that of the published work in [9] from 0 to 3 $\mathrm{THz}$, which theoretically models the desired performance for the $\mathrm{THz}$ device via an ADS circuit model.

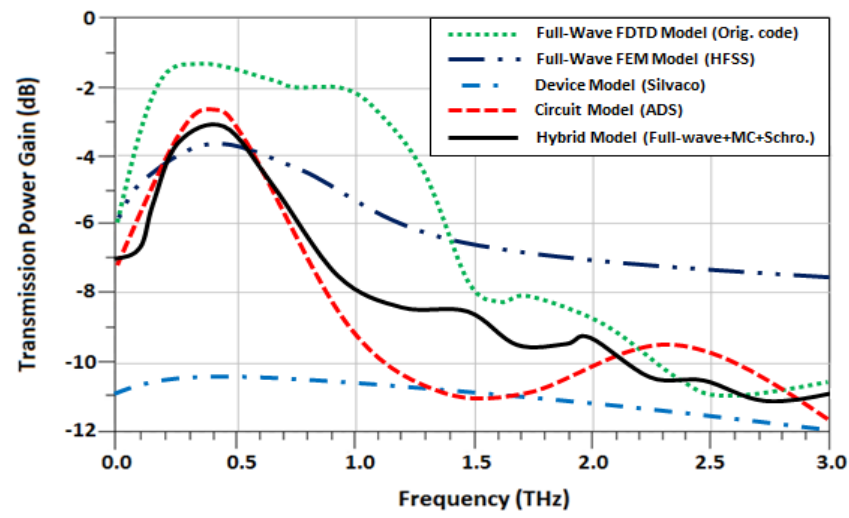

Fig. 8. Gain vs. frequency for a GaN HEMT. Curves are shown for: (a) standard full-wave time-domain analysis (FDTD), (b) commercial full-wave analysis (FEM), (c) commercial device model (Silvaco), (d) equivalent circuit model (ADS) found in Ref. [9], and (e) our proposed hybrid model with Schrodinger's equations.

With the addition of Schrodinger-corrected transport equations, the HEMT gain performance is seen to be closer to the ADS model prediction near the peak frequency of 0.5 THz. Fig. 8 also shows that the solution from commercial electromagnetic (ANSYS HFSS) and device automation packages (Silvaco) are not as accurate. Specifically, without the hydrodynamic model, resonances at higher frequencies are less prominent. Without an RTD, the gain remains below 0 $\mathrm{dB}$, but upon integration of the HEMT and RTD, a gain medium is created due to higher plasma wave group velocity than electron drift velocity.

To further compare these results, and ensure that the electron velocity and cutoff frequency are appropriate functions of the other parameters, obtained device values were compared with the published trends in Fig. 1 of ref [25] and Fig. 3 of ref [26]. For example, the peak drift velocity that we have calculated averages out to around $2.2 \times 10^{5} \mathrm{~m} / \mathrm{s}=2.2 \times 10^{7}$ $\mathrm{cm} / \mathrm{s}$. From the field profiles, we calculated the potential near the channel to be averaged to $0.5963 \times 10^{-6} \mathrm{~V}$. When dividing it by the barrier thickness, which in those simulations we had placed as $0.3 \mathrm{~nm}$, we calculate the E-field to be equal to approximately $1987.7 \mathrm{~V} / \mathrm{m}=198770 \mathrm{~V} / \mathrm{cm}=198.77 \mathrm{kV} / \mathrm{cm}$. We found this comparable to the results in ref [25], where the peak velocity appears at a field value of close to $200 \mathrm{kV} / \mathrm{cm}$, and has a value of $2.4 \times 10^{7} \mathrm{~cm} / \mathrm{s}$. In addition, Fig. 3 of ref [26] plots relations between $1 / \mathrm{L}_{\mathrm{G}}$ and the cutoff frequency. As explained in Fig. 1, the model for the HEMT consists of cascaded stages of $10 \mathrm{~nm}$ gate length devices, so using the relation of the $\mathrm{L}_{\mathrm{G}} / \mathrm{d}$ leads to a value of 0.667 at $1 / \mathrm{L}_{\mathrm{G}}=0.1 \mathrm{~nm}^{-1}$ if we consider the same value of $d$ as in the simulations. If we follow the trends of the linear $f_{T}$ vs. $1 / L_{G}$ curve, we realize that

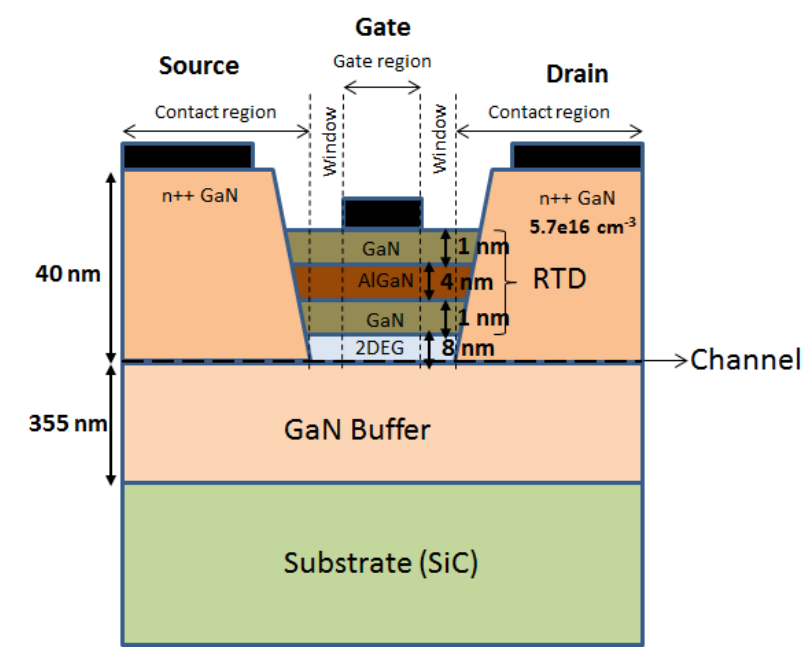

Fig. 9. Schematic of GaN RTD-Gated HEMT.

at the particular gate length, we can go up to $1.8 \mathrm{THz}$. However, the greater deviation from the linear $\mathrm{f}_{\mathrm{T}}$ curve upon decreasing gate length prevents the $\mathrm{f}_{\mathrm{T}}$ from reaching that value, and therefore corresponds to a frequency equal or lower than $1 \mathrm{THz}$.

The RTD consists of $\mathrm{AlGaN}$ and $\mathrm{GaN}$ layers and their thicknesses and dopings are given in Fig. 9. Different structures are then compared of the HEMT with and without the RTD, and the most optimum design is then evaluated for gain comparison. Fig. 10 depicts the gain performance of this particular structure. A HEMT with no RTD has a resonance at $250 \mathrm{GHz}$ with a potential gain of only $-1.05 \mathrm{~dB}$. By contrast, inclusion of the GaAs RTD shifts the resonance to as much as $2.25 \mathrm{THz}$ and has a gain of $3.75 \mathrm{~dB}$. Even better, changing the RTD material to $\mathrm{GaN}$ provides for greater gain, up to $6 \mathrm{~dB}$.

We remark that the gain increase for the RTD-gated HEMT is due to the negative differential conductance of the RTD. The latter serves to counteract damping and losses in the HEMT, implying enhanced responsivity. At the same time, there is a change in the gate-to-channel capacitance due to the geometrical features of the RTD tunnel layers. The smaller capacitance and resistivity tend to increase the resonant frequency and HEMT gain. Concurrently, the response becomes more narrowband. That is, there are tradeoffs

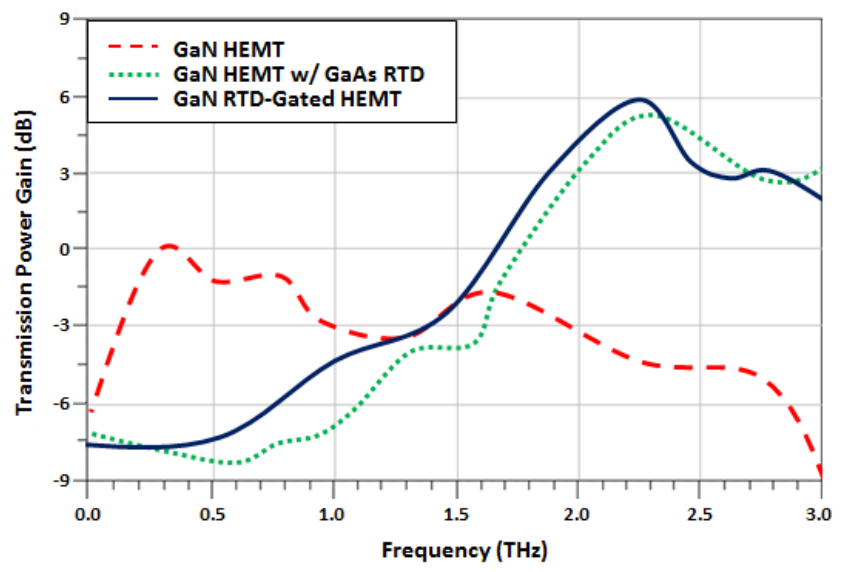

Fig. 10. Maximum Gain vs. frequency for a GaN RTD-Gated HEMT, GaAs RTD-Gated GaN HEMT, and conventional GaN HEMT. 
between the RTD-gated and simple HEMTs.

\section{CONCLUSION}

Though existing full-wave computational tools are accurate for lower frequency applications, there is a need to incorporate specific transport equations at the $\mathrm{THz}$ regime. This paper proposed the integration of Schrodinger's equations to provide more complete modeling of all full-wave effects including electron-wave interactions. Our improved simulations were compared with the published results that were based on distributed circuit models for validation. Our hybrid model was then used to demonstrate an increased gain for an RTDgated HEMT up to $3 \mathrm{~dB}$ and much higher resonant frequency, as much as $2.25 \mathrm{THz}$. These rigorous simulations show promise in physically realizing devices that can operate at high gain in the THz regime. Further optimizations and validations will be carried out in the future using fabricated and measured structures.

\section{ACKNOWLEDGEMENTS}

This work was supported by the Office of Naval Research N00014-11-1-0721, Devices and Architectures for THz Electronics MURI, Paul Maki program manager.

\section{REFERENCES}

[1] T. Nagatsuma, S. Horiguchi, Y. Minamikata, Y. Yoshimizu, S. Hisatake, S. Kuwano, N. Yoshimoto, J. Terada, and H. Takahashi, "Terahertz wireless communications based on photonics technologies," Opt. Express 21, pp. 23736-23747, 2013.

[2] S.A. Lynch, D. J. Paul, P. Townsend, G. Matmon, Z. Suet, R. W. Kelsall, Z. Ikonic, P. Harrison, J. Zhang, D.J. Norris, A.G. Cullis, C. R. Pidgeon, P. Murzyn, B. Murdin, M. Bain, H.S. Gamble, M. Zhao, and W. Ni, "Towards silicon-based lasers for terahertz sources," IEEE Journal of Selected Topics in Quantum Electronics, vol. 12, no. 6, pp. 1570-1578, 2006.

[3] M. Marso, "GaN for THz sources," Advanced Semiconductor Devices \& Microsystems (ASDAM), 2010 8th International Conference, pp.147154, 25-27 Oct. 2010.

[4] Y. Arakawa, "Progress in GaN-based quantum dots for optoelectronics applications," IEEE Journal of Selected Topics in Quantum Electronics, vol. 8, no. 4, pp. 823-832, 2002.

[5] R. Dingle, H.L. Stormer, A.C. Gossard, and W. Wiegmann, "Electron mobilities in modulation-doped semiconductor heterojunction superlattices," Appl. Phys. Lett., vol. 33, no. 7, pp. 665-667, July 1978.

[6] P.L. Solomon and H. Morkoc, "Modulation-doped GaAs/AlGaAs heterojunction field-effect transistors (MODFET's), ultrahigh-speed device for supercomputers," IEEE Trans. Electron Devices, vol. ED-31, no. 8, pp. 1015-1027, Aug. 1984.

[7] R. Dingle, M.D. Feuer, and C.T. Tu, "The selectively doped heterostructure transistor: materials, devices, and circuits" VLSI Electronics Microstructure Science, vol. 11, pp. 215-264, Academic Press, Orlando, 1985.

[8] S. Tenneti, N. K. Nahar, and J.L. Volakis, "Full-wave electromagnetic modeling of terahertz RTD-gated HEMTs," IEEE International Symposium on Antennas and Propagation, Lake Buena Vista, FL, July 7-12, 2013.

[9] B. Sensale-Rodriguez, L. Lei, P. Fay, D. Jena, and H. G. Xing, "Power amplification at $\mathrm{THz}$ via plasma wave excitation in RTD-gated HEMTs," IEEE Transactions on Terahertz Science and Technology, vol.3, no.2, pp.200-206, March 2013.
[10] B. Sensale-Rodriguez, P. Fay, D. Jena, and H. G. Xing, "Enhanced terahertz detection in resonant-tunnel diode gated HEMTs," ECS Transactions, vol. 49, no.1, pp.93-102, September 2012.

[11] M. Dyakonov and M. Shur, "Shallow water analogy for a ballistic field effect transistor: new mechanism of plasma wave generation by DC current," Phys. Rev. Lett. 71, pp. 2465-2468, 1993.

[12] V. Ryzhii and M. Shur, "Plasma instability and nonlinear terahertz oscillations in resonant-tunneling structures," Jpn. J. Appl. Phys., vol. 40, pp. 546-550, 2001.

[13] P. Burke, I. Spielman, J. Eisenstein, L. Pfeiffer, and K. West, "High frequency conductivity of the high-mobility two-dimensional electron gas," Appl. Phys. Lett., vol. 76, pp. 745-747, 2000.

[14] J. Torres, H. Marinchio, P. Nouvel, G. Sabatini, C. Palermo, L. Varani, L. Chusseau, P. Shiktorov, E. Storikov, and V. Gruzinskis, "Plasma waves subterahertz optical beating detection and enhancement in longchannel high-electron mobility transistors: experiments and modeling," IEEE Journal of Selected Topics in Quantum Electronics, vol. 14, no. 2, pp. 491-497, 2008.

[15] M.A. Alsunaidi, S.M.S. Imtiaz, and S.M. El-Ghazaly, "Electromagnetic wave effects on microwave transistors using a full-wave time-domain model," IEEE Transactions on Microwave Theory and Techniques vol.44, no.6, pp.799-808, Jun 1996.

[16] Y.A. Hussein, S.M. El-Ghazaly, and S.M. Goodnick, "An efficient electromagnetic-physics-based numerical technique for modeling and optimization of high-frequency multifinger transistors," IEEE Transactions on Microwave Theory and Techniques, vol.51, no.12, pp.2334-2346, Dec. 2003.

[17] S.M. Goodnick, S.S. Pennathur, U.A. Ranawake, P.M. Lenders, and V.J. Tripathi, "Parallel implementation of a Monte Carlo particle simulation coupled to Maxwell's equations," Int. J. Numerical Modeling: Electronic Networks, Devices and Fields, vol. 8, pp. 205-219, 1995.

[18] S.M.S. Imtiaz and S.M. El-Ghazaly, "Performance of MODFET and MESFET: a comparative study including equivalent circuits using combined electromagnetic and solid-state simulator," IEEE Transactions on Microwave Theory and Technology, vol. 46, no. 7, pp. 923-931, Jul. 1998.

[19] T. Otsuji, T. Watanabe, S.A. Boubanga Tombet, A. Satou, W.M. Knap, V. V. Popov, M. Ryzhii, and V. Ryzhii, "Emission and detection of terahertz radiation using two-dimensional electrons in III-V semiconductors and graphene," Terahertz Science and Technology, IEEE Transactions on , vol.3, no.1, pp.63-71, Jan. 2013.

[20] M. Ghiamy, A. Abdipour, and R. Moeeni, "Field analysis (full-wave) of millimeter-wave HEMTs," Microwave Electronics: Measurements, Identification, Application Conference 2001, pp.121-126.

[21] G. Mur, "Absorbing boundary conditions for the finite-difference approximation of the time-domain electromagnetic-field equations," IEEE Transactions on Electromagnetic Compatibility, vol.EMC-23, no.4, pp.377-382, Nov. 1981.

[22] M. A. Khorrami, S. El-Ghazaly, S. Yu, and H. Naseem, "Analytical modeling of terahertz wave propagation inside ungated two-dimensional electron gas layers," 2011.

[23] A. Ashok, D. Vasileska, O.L. Hartin, and S.M. Goodnick, "Electrothermal Monte Carlo simulation of GaN HEMTs including electron-electron interactions," IEEE Transactions on Electron Devices, vol.57, no.3, pp.562-570, March 2010.

[24] B. Winstead and U. Ravaioli, "A quantum correction based on Schrodinger equation applied to Monte Carlo device simulation," IEEE Transactions on Electron Devices, vol. 50, no. 2, February 2003.

[25] S. Yamakawa, S. Goodnick, S. Aboud, and M. Saraniti. "Quantum corrected full-band cellular Monte Carlo simulation of AlGaN/GaN HEMTs," Journal of Computational Electronics 3, pp. 299-303, 2004.

[26] D. Guerra, R. Akis, D. K. Ferry, S. M. Goodnick, and M. Saraniti. "Cellular Monte Carlo study of RF short-channel effects, effective gate length, and aspect ration in GaN and InGaAs HEMTs," Computational Electronics (IWCE), $201014^{\text {th }}$ International Workshop on, pp. 26-29, Oct. 2010. 

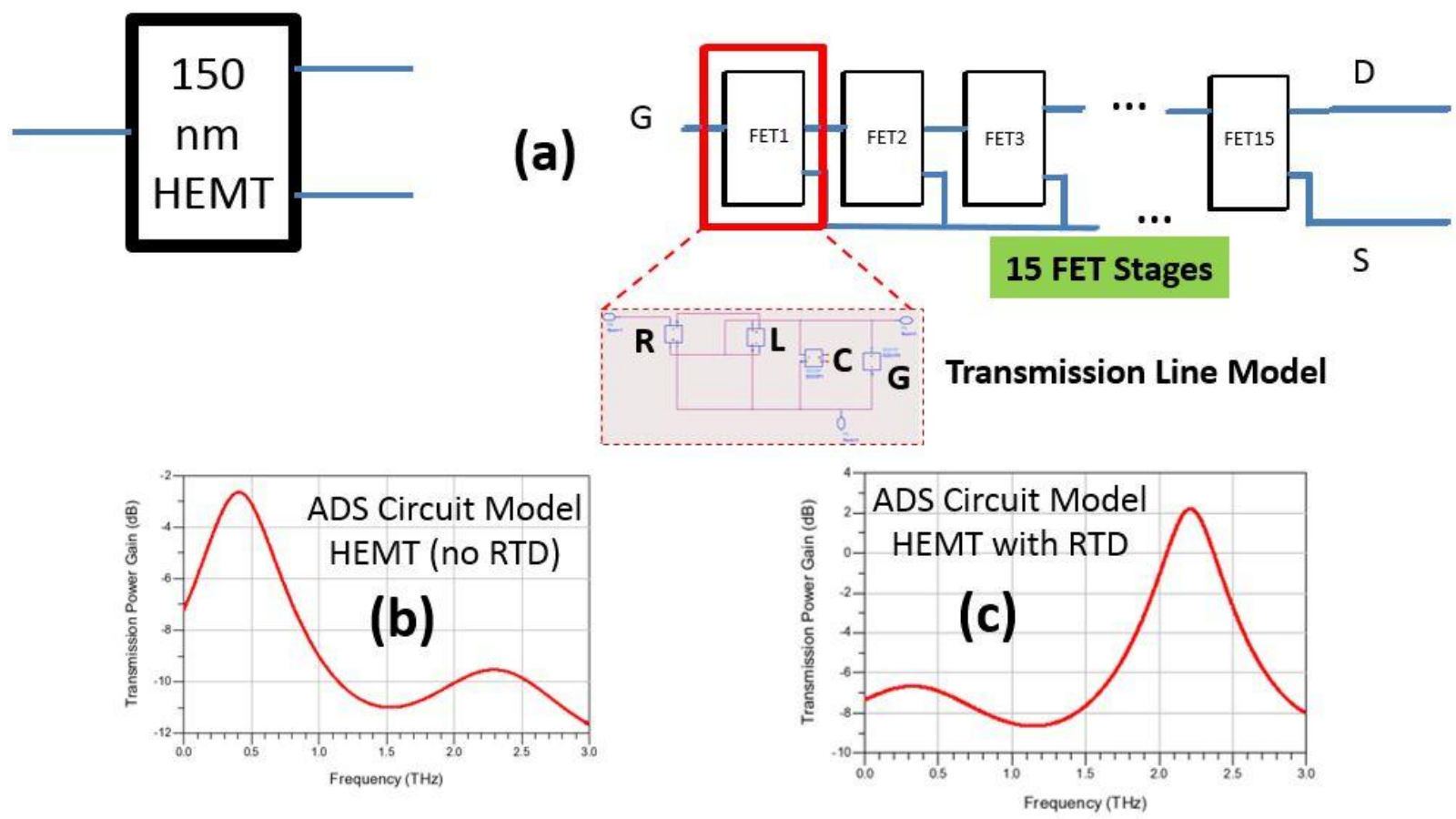